\title{
DECAMETER PULSARS AND TRANSIENTS SURVEY OF THE NORTHERN SKY. STATUS, FIRST RESULTS, MULTIPARAMETRIC PIPELINE FOR CANDIDATE SELECTION
}

\author{
V.V.Zakharenko ${ }^{1}$, I.P.Kravtsov ${ }^{1}$, I.Y.Vasylieva ${ }^{1,2}$, S.S.Mykhailova ${ }^{3}$, O.M.Ulyanov ${ }^{1}$, \\ A.I.Shevtsova ${ }^{1}$, A.O.Skoryk ${ }^{1}$, P.Zarka ${ }^{2}$, O.O.Konovalenko ${ }^{1}$ \\ ${ }^{1}$ Institute of Radio Astronomy of the National Academy of Sciences of Ukraine (IRA NASU) \\ 4, Chervonopraporna St., 61002, Kharkiv, Ukraine, rian@rian.kharkov.ua \\ ${ }^{2}$ LESIA, Observatoire de Paris, CNRS, UPMC, Universite Paris Diderot, Meudon, France \\ ${ }^{3}$ V. N. Karazin Kharkiv National University, 4 Svobody Square, 61022, Kharkiv, Ukraine, \\ info@karazin.ua
}

ABSTRACT. We present the results of processing first $20 \%$ of Northern sky pulsars and transients survey using UTR-2 radio telescope. Data processing is done by an automatic pipeline that detects and outputs a large number of transient candidates (usually dispersed bursts). We have developed a multivariate pipeline for visual inspection of these candidates. By adjustment of input parameters of the pipeline the observer can substantially increase signal-to-noise ratio of detected signals as well as discriminate them from residual low-intensity interference with high significance. About 450 transient signals have passed the examination by the multivariate pipeline. Their distributions on the Galactic latitude and dispersion measure have been derived. The shape of the distributions suggests that these signals might be associated with cosmic sources of radio emission.

Keywords: pulsars, transients, survey, decameter range

\section{Introduction}

First decameter-wavelength census of known pulsars (Zakharenko et al., 2013) aimed at re-detection and study of nearby pulsar population was highly successful. More than a half of observable pulsars with dispersion measure (DM) $<30 \mathrm{pc} \mathrm{cm}^{-3}$ were re-detected, despite the extremely difficult conditions of observations in the decameter range, such as high Galactic background temperature, large scattering time constant of pulsar pulses, enormously high radio frequency interference (RFI) level etc. The major result of that study was confirmation of pulse profile broadening observed for the majority of aforementioned pulsars, compared to their widths at higher observational frequencies. This opened a new parameter space for searching new sources of pulsed radiation in the closest Galactic neighborhood. Pulse profile broadening can make 'unfavourably' oriented pulsars, rotating radio transients (RRATs) and other radio-emitting neutron stars visible at low frequencies. This makes a low-frequency blind pulsar and transient survey of the entire Northern sky both useful and required.

Such a survey was started in 2010 (Zakharenko et al., 2011). One of the first important results was first detection of decameter emission (Vasylieva et al., 2014) of the re- cently discovered pulsar J0243+6257 (Hessels et al., 2008). The dispersion measure of this pulsar was substantially refined (an error became less than $0.01 \mathrm{pc} \mathrm{cm}^{-3}$ instead of previous one near 0.2). It became possible due to the important advantage of the low-frequency range and broad observational band $(16.5-33 \mathrm{MHz})$ of the above survey - the precise determination of the dispersion measure. Observations in a broad frequency band allow to distinguish with high confidence between a real signal that had passed through the interstellar medium and local interference. The real signals have a characteristic dependence of pulse arrival time on the frequency $\left(\propto f^{-2}\right)$, being delayed at lower frequency $\mathrm{f}_{\min }$ compared to higher frequency $\mathrm{f}_{\max }$ by

$$
d t=10^{16} / 2.410331 \cdot \mathrm{DM} \cdot\left(f_{\min }^{-2}-f_{\max }^{-2}\right)
$$

seconds. The local signals may have a different origin and time-frequency structure, e.g. signals with linear frequency modulation.

Stream processing of a part of survey data revealed many transient 'candidates'. The goal of the present work is to check each 'candidate' by means of the interactive data reduction pipeline, all intermediate results of which can be inspected simultaneously. For instance, raw dynamic spectra (before de-dispersion) show whether signal's time-frequency shape is not $\propto f^{-2}$ (Vasylieva et al., 2014) or has features similar to those described in (Petroff et al., 2015). Presence of signal only in a narrow frequency band is also an indicator of its artificial origin. However, as the signal-to-noise ratio (SNR) of the candidates after integration of the entire frequency band is quite low, generally 6-8 standard deviations $(\sigma)$, inspection in the fractional sub-bands reduces it further, sometimes equalizing signal with noise. Therefore, additional criteria are required to verify the signals' origins. Subsequent stage of our work is an analysis of distribution of the 'candidates' on the galactic coordinates and dispersion measures (that can be translated to distance). Similarity of these distributions to distributions of known pulsars and RRATs will imply the cosmic origin of the 'candidates'. 


\section{Data analysis}

The blind decameter drift-scan survey (Zakharenko et al., 2011; Vasylieva et al., 2014) should cover a range of declinations $(\delta)$ between $-10^{\circ}$ and $90^{\circ}$. To date, more than $90 \%$ of planned sky region is covered, and about $75 \%$ of data is automatically pre-processed (i.e. spared from interference, compensated for interstellar dispersion in the range $0<\mathrm{DM}<30 \mathrm{pc} \mathrm{cm}^{-3}$ with $0.01 \mathrm{pc} \mathrm{cm}^{-3}$ step, producing 3000 spectral-integrated time series that form a "DM-Time" plane, see Fig. 1a). After processing of each observation session, the database of candidates is updated with parameters (occurrence time, DM and signal-to-noise ratio) of detected signals if their SNR exceeds 5.5 $\sigma$.

At the next stage signals with $\mathrm{DM}<1$ and signals which form regular structures (usually slanted lines) in the "DM-Time" plane are rejected as they are most likely RFI. Taking into account scattering in the interstellar medium that smears pulses of regular pulsars over tens of milliseconds and pulse width itself, we also reject the events that occupy only a single sample $(8 \mathrm{~ms} \times 0.01 \mathrm{pc}$ $\mathrm{cm}^{-3}$ ) in the "DM-Time" plane.

In order to reveal spurious (RFI-induced) candidates, and to maximize the SNR of real candidates, we have developed a multivariate pipeline for candidate selection. A set of adjustable input parameters includes:

- thresholds in RFI mitigation routine (Vasylieva et al., 2013),

- step between adjacent trial DM values (up to $0.002 \mathrm{pc} \mathrm{cm}^{-3}$ ),

- cutoff frequency of a high-pass filter that reduces effects of ionospheric scintillations.

- cutoff frequency of a low-pass filter that increases an integration time for the pulses

- width of partial frequency sub-bands

- time interval for search of repetitive signals

When optimal, the above settings lead to 2-5-fold increase in SNR which is vital for exploring spectral features of candidates and their shape in the 'DM-Time' plane with a higher contrast.

Fig. 1 shows visual inspection of a 'candidate' that at first glance didn't provide any reason for rejection (a red disk in the panel a). However, after examining the raw data and its de-dispersion using the correct DM value, we found a signal behavior, very different from that described by equation (1). The signal was relatively narrowband (panel b) and had a linear frequency modulation (panel c, top). Its shape in the time-DM plane is smeared over many adjacent DM values (panel c, bottom) as it happens with RFI signals. Signals like in Fig. 1 are of course excluded from 'candidates' database.

\section{Results and discussion}

As a result, 'candidates' from about $20 \%$ of the survey have been checked for compliance with the dispersion law (equation 1). These signals have a relatively broad band and don't show any RFI features. We determined approximate coordinates of each 'candidate' by deriving direction of UTR-2 pointing at the moment of its detection.

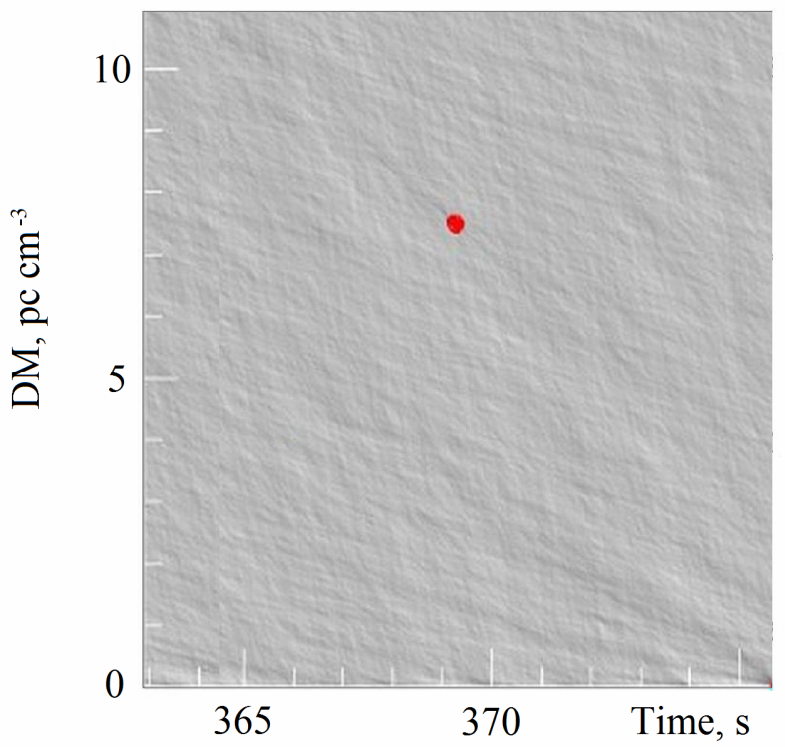

a)

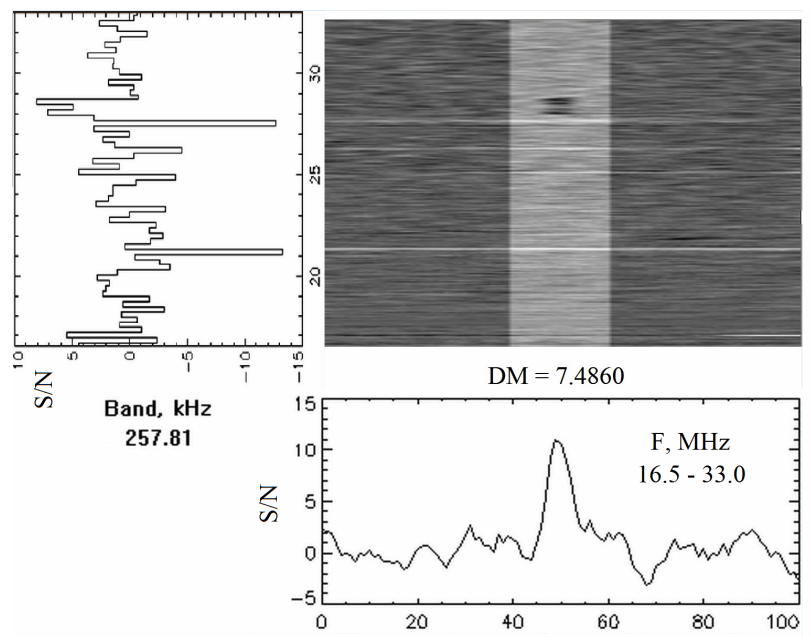

b)
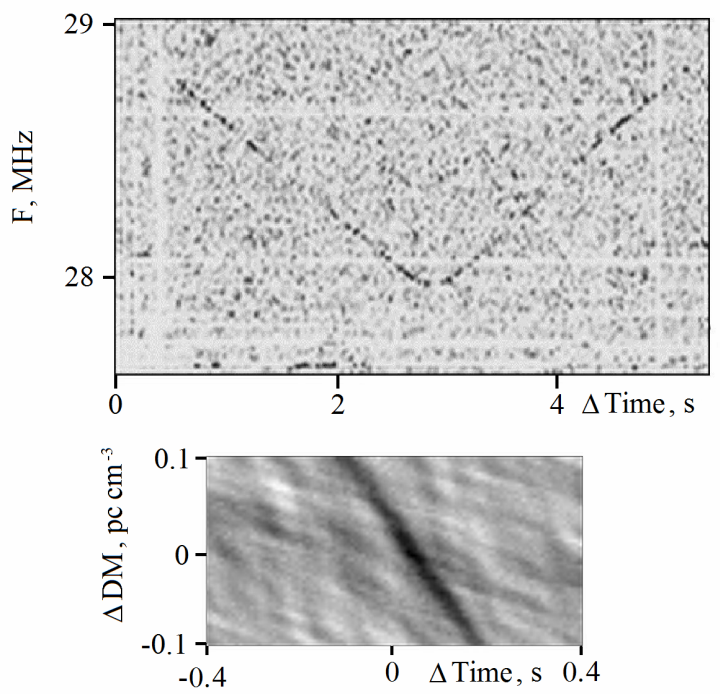

c)

Figure 1: (a) "DM-time" plane, (b) de-dispersed spectrogram (grey-scale image) with cut-sets over time and frequency, (c) zooms of raw spectrogram (top) and "DMtime" plane (bottom) 
A map of Galactic background radio emission (Sidorchuk et al., 2008) and superimposed coordinates of the corresponding transient sources are shown in Fig. 2. Only recordings of low declinations are (partly) processed so far.

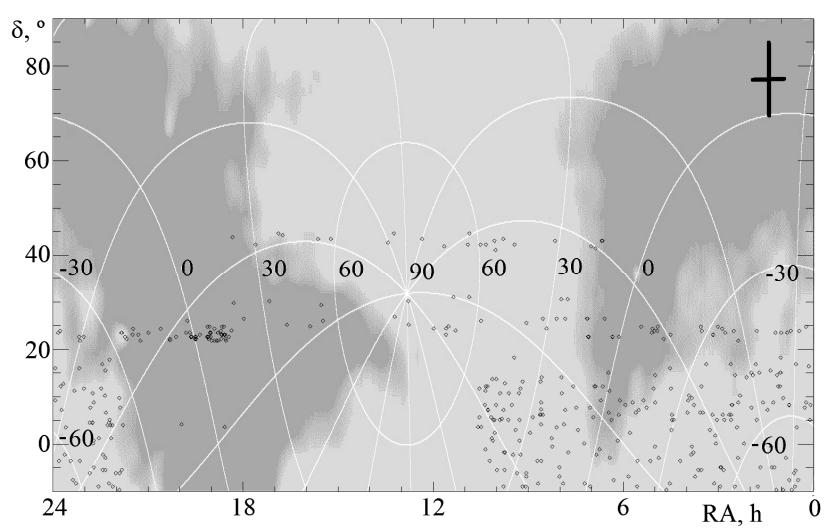

Figure 2: Sky map with coordinates of transient candidates and pattern of directivity of the UTR-2

A promising result is a lack of preferable directions in the distribution of the sources, except for a region of $\mathrm{RA}=19^{\mathrm{h}}$ and $\delta=23^{\circ}$. But as this region is located close to Galactic disk, in the direction not far from its center, this concentration of detected events seems expected.

To analyze statistically whether the above sources are a part of Galactic disk population, we have derived a histogram of their galactic latitudes. As expected, the histogram has a local maximum near $b=0^{\circ}$ (Fig. 3 a), similar to the histogram for close pulsars (period $>0.2 \mathrm{~s}, \mathrm{DM}<30$ pc $\mathrm{cm}^{-3}$ ), shown in Fig. 3b (derived from Manchester et al., 2005).

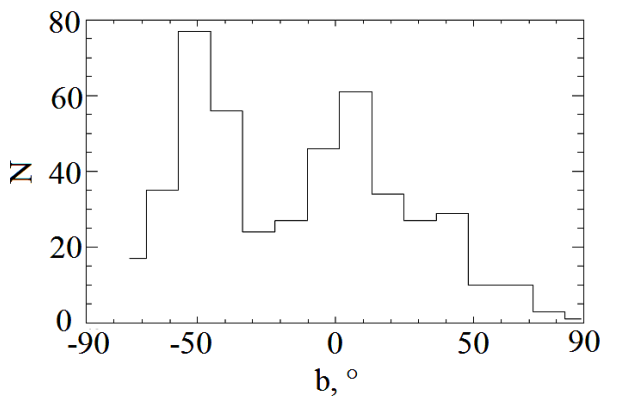

a)

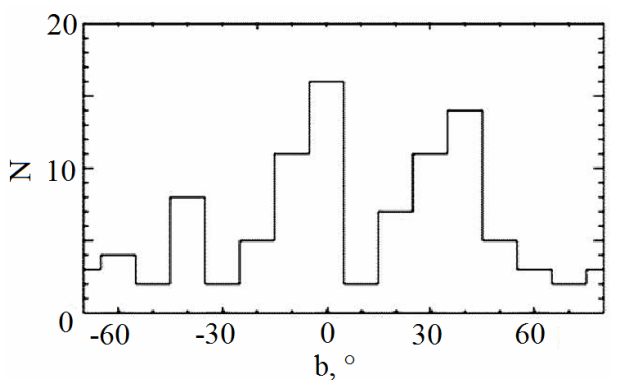

b)

Figure 3: Histogram of transients' (a) and known close pulsars' (b) (Manchester et al., 2005) galactic latitudes
The main maximum of the histogram is at $b=-50^{\circ}$. But from Fig. 2 it is evident that the data with galactic latitude from $-45^{\circ}$ to $-90^{\circ}$ is processed completely, whereas the remaining areas are processed partly. Therefore, along with further data processing the number of events at galactic latitudes above $-45^{\circ}$ will increase, while below $-45^{\circ}$ will remain unchanged. Furthermore it is possible that at low radio telescope beam positions (corresponding to low declinations in Fig. 2 and regions with $-90^{\circ}<\mathrm{b}<-45^{\circ}$ ) the number of residual low-intense broadband RFI in the data is higher.

The distribution on the galactic latitude thus implies that if not all, but the majority of detected signals are of cosmic origin and their coordinates tend to low Galactic latitudes. It is possible that there is a number of interference among the 'candidates', but they do not have a large impact on the shape of the distribution.

The expected distribution of cosmic sources on DM is shaped by the following factors. With increasing DM (and therefore distance between a source and an observer) the geometrical size of the considered region (layer), and therefore the number of sources that it contains, should increase. For the closest ones (while the considered layer doesn't extend beyond the width of the Galactic disk) the number of sources grows approximately as a square of distance (and hence of DM). For more distant sources the increase will be close to linear. However at some point this distribution will reach the maximum and the number of sources will start to decrease, because the more distant sources are generally weaker. Additionally, with decreasing frequency, the scattering of pulsed signals comes into play (the scattering time constant is $\propto \mathrm{f}-4$ ). I.e. at lower frequencies this turnover should happen at lower values of DM, compared to higher frequencies.

For comparison, a simulation of similar distributions for pulsars, expected to be detected by SKA1-LOW and SKA1-MID is shown in Fig. 4 (Keane et al., 2015). A distribution, obtained for the above 'candidates' is shown in Fig. 5a, and is generally in agreement with the simulated one (Fig. 4), as we see the increase in number of 'candidates' up to $\mathrm{DM}=10-15$, followed by a gradual decrease.

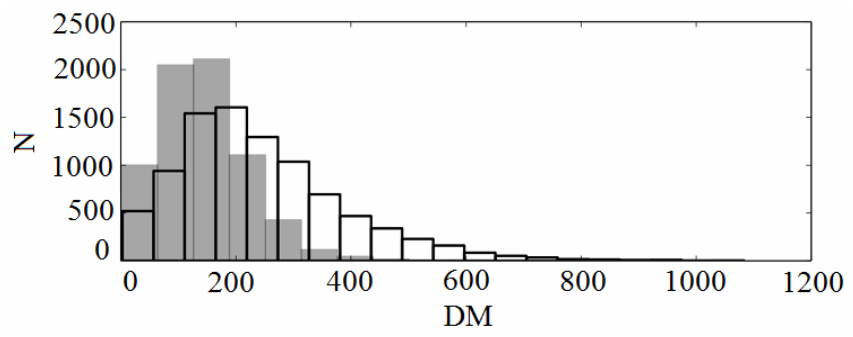

Figure 4: Simulated histograms of DM of pulsars, expected to be observed with SKA Phase 1, for SKA1-LOW (dark bars) and SKA1-MID (clear bars) (Keane et al., 2015)

Apart from an outlier at a low DM value (that can be related either to RFI or to unidentified source of repetitive pulsed emission, e.g. a pulsar) the distribution is smooth. For comparison, Fig. 5b shows a distribution of known 
RRATs on dispersion measure, derived from 'RRATalog'. It has a fragmented structure due to a small number of sources, but as a whole, it is also similar to those shown in Fig. 4 and Fig. 5a. We should note that the shape of RRAT distribution is a result of their discoveries in highfrequency surveys - at decimeter wavelengths, which means the lower influence of the interstellar medium and a larger DM range that can be explored.

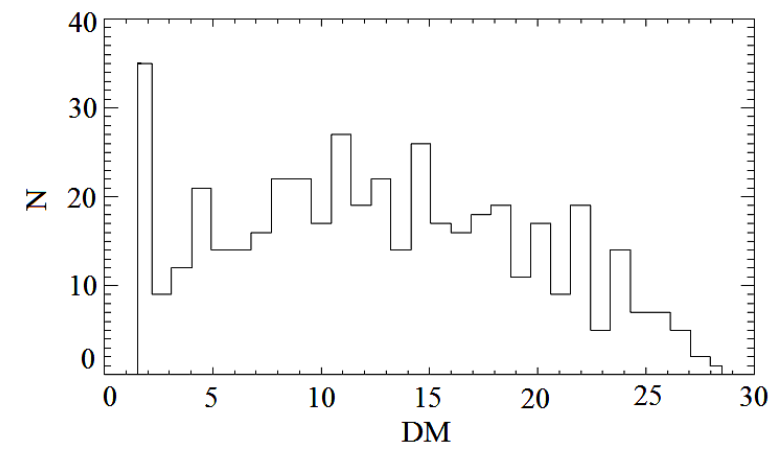

a)

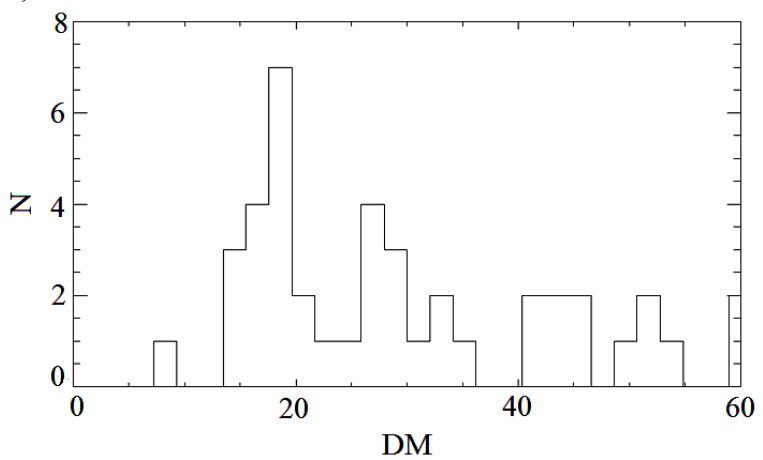

b)

Figure 5: Distribution of DM of transient events detected in this study (a) and known RRATs (b) (RRATalog)

Thus, the both distributions that we derived (on the galactic latitude and DM) suggest that the majority of detected signals are most likely of cosmic origin. The shape of the received pulses is similar to pulsar or RRAT pulses. We assume that the detected 'candidates' could originate from:

- neutron stars that are unfavorably oriented in space for higher frequency observations,

- RRATs with low dispersion measures that have been misinterpreted as RFI in the high-frequency surveys,

- pulsars that present an increase of flux density towards low frequencies (such as PSR B0943+10, see Stappers et al., 2011),

- giant pulses of millisecond pulsars (usually flux density of millisecond pulsars grows with decreasing frequency) that are either sporadic or unfavorably oriented. Although giant pulses are short, they will arrive significantly scattered at our frequencies and will be noticeable in the survey data,

- anomalously intense pulses (see Ulyanov et al, 2006) of close weak pulsars.

\section{Conclusions}

Using the developed routines of multivariate candidate selection, we have analyzed the transient signals from about $20 \%$ of the decameter pulsars and transients survey of northern sky. Adjustment of filtering parameters and simultaneous access to all intermediate stages of processing serves for rejection of RFI-induced candidates even with low SNR.

Histograms of candidates' galactic latitudes and dispersion measures show that corresponding sources tend to be located at low Galactic latitudes and have an expected distribution on dispersion measure. This allows us to suppose that the majority of above signals must be associated with cosmic sources.

Complete processing of all survey data give more significance to these distributions and possibly will allow to link such parameters as galactic longitude, intensity, probability of occurrence etc. This will help to attribute the sources to certain structures, such as Gould belt, Galactic spiral arms and subsequently to improve general knowledge of close neutron star population.

\section{References}

Zakharenko V.V. et al.: 2013, MNRAS, 431, 3624.

Zakharenko V.V. et al.: 2011, Odessa Astron. Publ., 24, 117. Vasylieva I.Y. et al.: 2014, $R P \& R A, 19,197$.

Hessels J.W.T. et al.: 2008, AIP Conf. Ser., 983, 613.

Petroff E. et al.: 2015, MNRAS, 451, 3933.

Vasylieva I.Y. et al.: 2013, Odessa Astron. Publ., 26, 159. Sidorchuk M.A. et al.: 2008, Astrophysics with E-LOFAR, http://www.hs.unihamburg.de/DE/Ins/Lofar/lofar_work shop/poster abstracts.html.

Manchester R.'. . et al.: 2005, $A J, \mathbf{1 2 9}, 1993$.

RRATalog: http://astro.phys.wvu.edu/rratalog

Keane E. et al.: 2015, AASKA14, 1, 40.

Stappers B.W. et al.: 2011, $A \& A, \mathbf{5 3 0}, \mathrm{A} 80$.

Ulyanov O.M. et al., 2006, IAU Joint Discussion, 2, 12. 